\title{
The Factors Influencing Fatigue Related to the Accident of Intercity Bus Drivers in Indonesia
}

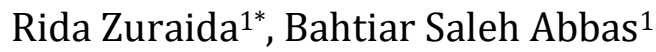 \\ ${ }^{1}$ Department of Industrial Engineering, Faculty of Engineering, Bina Nusantara University, Jakarta \\ Indonesia 11480
}

\begin{abstract}
As a developing country, Indonesia pursues advancement through rapid infrastructure expansion. However, in the transportation sector, safety is still a serious problem and requires attention. Road accidents in Indonesia with a high fatality mostly involve intercity buses. Most of the accidents are reportedly caused by human error, typically caused by a fatigued driver. To understand the factors that influence general fatigue (F), three variables-subjective workload (WL), need for recovery (NR), and emotional intelligence (EI) - are discussed by considering time on task and time of day as factors. To assess the perceptions of 298 intercity bus drivers, this research was conducted using a questionnaire. There are statistically significant differences in the NR $(p=0.001)$ and EI level $(p=0.001)$ among the time on task group (less than three hours, three to five hours, and longer than five hours) but not for the WL. Meanwhile, for the time of day group (driving in the morning, afternoon, and at night) there are no significant differences of WL ( $p=$ $0.161)$, NR ( $p=0.795)$, and EI level ( $p=0.271)$. A Tukey post hoc test revealed that the NR and is statistically significantly higher for a duration longer than three hours $(p=0.025$ and $p=0.000)$. Binomial logistic regression was run to understand the influence of $\mathrm{WL}, \mathrm{NR}$, and EI on subjective fatigue level, categorized into fatigue (1) and alert (0). The Hosmer-Lemeshow test showed that the model fit the data well, $p=0.673$. The variables NR $(p=0.08)$ and EI $(p=0.020)$ statistically significantly predict general fatigue subjectively. Based on these results, EI and NR are suggested as factors that should be analyzed further concerning the issue of fatigue-related accidents. Both factors should also be considered in company's fatigue management system.
\end{abstract}

Keywords: Emotional intelligence; Fatigue; Intercity bus drivers; Need for recovery; Workload

\section{Introduction}

The infrastructure in Indonesia is developing rapidly, and this includes the construction of toll roads, which encourage goods distribution and people to travel across the land. Today, Indonesian citizens and entrepreneurs have other transportation options, such as buses and travel cars, which take less time than before as an advantage of the availability of new toll road routes. This advantage, of course, must be followed by increasing safety aspects such as the availability of safety supporting tools/facilities (signs, guardrail, supervision by toll officer, and other supporting programs). Currently, the rate of traffic accidents in Indonesia is still high, including those involving buses and four-wheeled vehicles. In 2018, there were 550 bus and 6,892 car accidents (both toll and non-toll) (Polri, 2019). 
The accidents involving buses have the potential to generate more casualties, in terms of both injuries and deaths, than smaller vehicles. Bus accidents are considered important in various countries, such as Malaysia and China (Mohamed et al., 2012; Sang and Li, 2012). Some efforts to improve traffic safety to reduce the rate of accidents continue to be carried out by the Indonesian government. It is believed that safety can be achieved through individual approaches based on basic human values and risk perception (Sutalaksana et al., 2019). Thus, understanding what values and how risk is perceived by people should become the foundation for developing any traffic safety program.

Traffic accidents have long been the subject of extensive research in Indonesia, and it becomes a highly important factor in identifying discrepancies in traffic management and the entire transportation system (Jusuf et al., 2017). The common consensus recognizes that traffic accidents are the result of three different factor types: human, vehicle, and external factors (including road conditions), with human factors having the strongest influence (Soehodho, 2017). Concerning human aspects, fatigue plays an important role in traffic accident risk, and the relationship between fatigue and traffic accident risk is a major aspect that must be considered to improve safety (Williamson et al., 2011). Fatigue is generally defined as physiological and biochemical changes in the body, muscles, central nerves, hormones, and blood (Phillips, 2015). Fatigue is also defined as a decrease in ability and performance characterized by reduced attention, perceptions, decision making, and operational capabilities (Toomingas et al., 2012; Hirshkowitz, 2013; Phillips, 2015). These performances could lead to unsafe driving behavior that plays a role in traffic accidents, such as overtaking, ignoring the speed limit, driving errors, aggressive behavior, and others (Wedagama and Wishar, 2018). The literature concluded that fatigue is related at least to three aspects: physiological, objective, and subjective aspects.

Some studies that explore the factors contributing to driver fatigue note several important factors, such as time on task, time of day, primary and secondary workload, demographic aspect, environment, road conditions, and others (Di Milia et al., 2011; Fergusin et al., 2012; Friswell and Williamson, 2013; Hirshkowitz, 2013; Phillips, 2015; Chen and Zhang, 2016). Some of this research reports a changing pattern in fatigue indicators that did not consistent with others findings (Craig et al., 2012). In other words, how fatigue define and its level cause by driving job still has gaps, including influential factors.

It has been agreed that workload influences fatigue. During a driving task, a high workload led to excessive fatigue, as did a low workload, particularly one featuring monotony (May and Baldwin, 2009). Sleep deprivation also induces a kind of fatigue categorized as sleep-related fatigue, while that related to workload is categorized as taskrelated fatigue. Based on these classifications, the perception of workload should be included in studies on fatigue. Understanding whether drivers have the potential for sleeprelated fatigue is usually measured by the level of sleepiness before a driving task. However, risk likelihood scoring suggests assessing working conditions for the driver to get enough sleep/rest, driving duration, and night driving (Dawson et al., 2018).

Other factors may lead to fatigue, as humans are individually unique. The selfperceptions on certain condition can be influenced by intrinsic factors, such as emotional intelligence (EI). Therefore, some research suggests the role of emotions in cumulative fatigue (Kulik, 2011). EI includes skills of living, the abilities for empathy and understanding oneself, and the ability to build positive relationships with others (Kulik, 2011). The role of EI in cumulative fatigue is defined by its relation to the ability to cope with stress and adaptation to a certain condition (Noorbakhsh et al., 2010). 
Driving as a job can be stressful and it can boost fatigue levels, which may lead to an increased risk of accidents. Despite the fact that the rate of traffic accidents in Indonesia is still high, the research that explores the ability to cope with stress and adaptation is limited. In addition, researchers have also noted the correlation between fatigue and bus accidents (Mohamed et al., 2012; Sang and Li, 2012). However, EI as a contributing factor, along with other factors, is still not discussed. This study is a response to this gap because it measures the relationship of EI and other factors with bus driver fatigue. Compared to previous studies, this research incorporates the EI variable on the issue of fatigue-related accidents among intercity bus drivers. The following questions will be answered in this study: (1) what is the level of subjective workload, fatigue, need for recovery, and EI status of intercity bus drivers in Indonesia; and (2) what are the most relevant factors related to the fatigue of intercity bus drivers by considering time on task and time of day? The contributions of this study are: (1) providing a new perspective on the factors leading to fatigue; and (2) helping to understand the association of the fatigue among intercity bus drivers and influence factors to fatigue.

\section{Methods}

\subsection{Research Instrument}

A list of questions was developed to collect data for this study. A questionnaire measured the self-perception of workload, subjective fatigue level, need for recovery, and EI. All respondents are male and have a driver's license. For data collection, all respondents completed the questionnaire under the supervision of a survey officer to ensure they understood the questions.

The questionnaire consisted of workload and fatigue variables that were assessed by 10 questions each. The need for recovery variable was covered by 16 questions (Dawson et al., 2018), while the EI variable was examined using 25 questions (Wulandari, 2011). On the other hand, subjective fatigue level was measured using the Fatigue Assessment Scale (FAS) questionnaire (De Vries et al., 2003). In addition, the internal consistency or reliability of the instrument was measured using Cronbach's alpha, the value of which was 0.712, indicating a high level of internal consistency for the scale with this specific sample.

The fatigue questionnaire assessed tired feelings during the day (F1, F2, F3, F5, F9), signs of fatigue (F6, F7, F8), and energy during the day (F4 and F10), while the workload questionnaire assessed perceptions of physical workload (WL1, WL5, and WL10), mental stress (WL2, WL4, WL8, and WL9), monotony (WL3), and time management (WL6). Questions related to the need for recovery variable focused on hours of sleep and sleep adequacy before driving (NR1-NR3), activity hours before driving (NR4-NR6), driving duration (NR7-NR8), average rest hours before driving (NR9-NR11), average hours of night driving (NR12-NR13), and possible number of days off (NR14-NR16). Meanwhile, the questions related to the EI variable asked drivers about self-awareness (EI1-EI3, and EI9), self-regulation (EI4-EI8), social skills (EI10-EI13), empathy (EI14-EI20), and motivation (EI21-EI25).

To identify differences between the time on task and time of day groups in terms of Workload (WL), Fatigue (F), Need for recovery (NR), and EI, a statistical test using a oneway analysis of variance (ANOVA) was conducted. A logistic regression was applied to model the probability of driver fatigue with two conditions: alert (0) and fatigue (1). In this case, binary logistic regression is the mathematical approach used to analyze the relationship between one or more predictor variable and a response variable with dichotomous or binary categories. In general, the mathematical model of logistic regression is as follows (Agresti, 2002): 


$$
\pi(x)=\frac{e \beta_{0}+\beta_{1} X_{1}+\beta_{2} X_{2}+\cdots+\beta_{k} X_{k}}{1+e \beta_{0}+\beta_{1} X_{1}+\beta_{2} X_{2}+\cdots+\beta_{k} X_{k}}
$$

where $\pi$ is the binary outcome, $\beta$ is the model parameter, and $X$ is the predictor variable.

Model testing was conducted using the Hosmer-Lemeshow test (Hosmer and Lemeshow, 2000; Sperandei, 2014), and goodness of fit was measured using the chi-square value at a significance level of $\alpha=5 \%$. If the Hosmer-Lemeshow goodness of fit value is $\leq$ 0.05 , there will be significant differences between the model and observation result, but if the value is $>0.05$, it indicates that the model is able to predict the observation result.

\subsection{Sample Size}

About 300 male bus drivers agreed to complete the questionnaire. However, two questionnaires were excluded due to incompleteness. Thus, the data of 298 questionnaires were analyzed in this study. According to the Cochran formula, the number of participants was sufficient for the research. The Cochran formula is commonly used to determine the adequacy of the samples in survey research (Bartlett et al., 2001), and it was calculated as follow:

$$
n=\frac{z^{2} p(1-p)}{d^{2}} ; n=\frac{1.645^{2} \times 0.5 \times(1-0.5)}{0.1^{2}}=271 \text { data }
$$

The value of $z$ is the critical value for the desired level of confidence, $p$ is the proportion of certain characteristics in the population, and $d$ is the level of accuracy. For large and unknown population sizes, the maximum variability assumption is used, so the $p$-value is set at 0.5 .

\section{Results and Discussion}

\subsection{Descriptive Data}

From 300 obtained questionnaires, only 298 were processed for analysis. The bus drivers came from nine bus companies. The respondents' ages were between 25 and 68 years ( $44.9 \pm 9.75$ years) with one to 20 years of experience as a driver (12.28 \pm 8.9 years). Duration of a one-way trip was reported as less than three hours by $13.3 \%$ of drivers, three to five hours by $55.1 \%$ of drivers, and more than five hours by $26.6 \%$ of drivers. Meanwhile, related to distance, $23.7 \%$ of drivers needed to drive over $350 \mathrm{~km}$ each trip, and rests were only between every 120 and $350 \mathrm{~km}$. About $66 \%$ of drivers were assigned an hourly schedule from 12:00 to 18:00, while 6\% of drivers were assigned to drive before 09:00 and $8 \%$ of drivers were assigned to drive from 09:00 h to 12:00, and rests (about 20\%) were assigned after 18:00.

\subsection{Questionnaire Results}

Data collected from participants for each variable were plotted in some graphics grouped by time on task (less than three hours, three to five hours, and more than five hours) and time of day (morning, afternoon, and night). Figure 1 below shows the result of workload variable based on time on task. Drivers' workload grouped by time on task and time of day appears similar graphically, although the workload of drivers who drive less than 3 hours or drive in the morning seems a little higher than others. On the other hand, the questionnaire result of the fatigue variable grouped by time on task and time of day is plotted in Figure 2. 


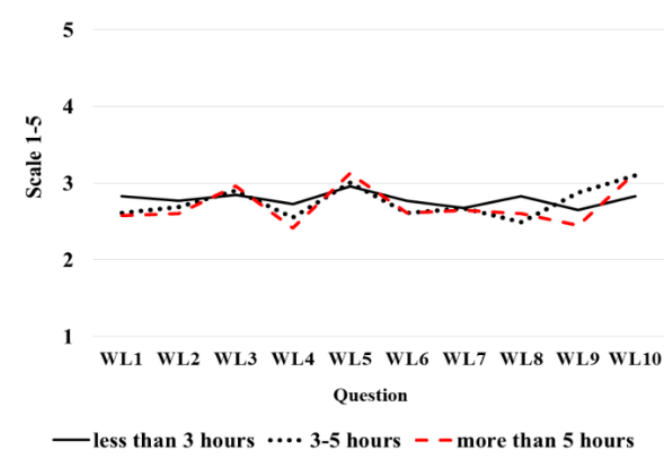

(a)

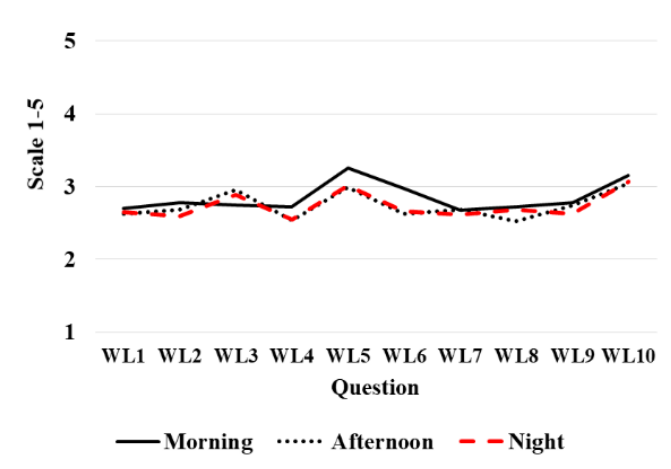

(b)

Figure 1 Questionnaire result of workload variables

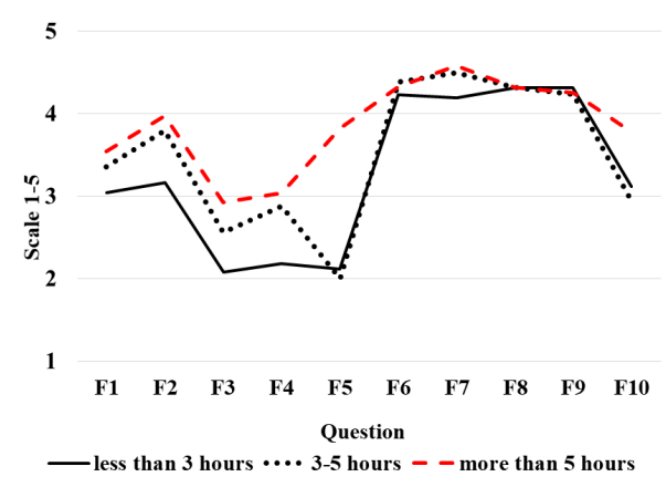

(a)

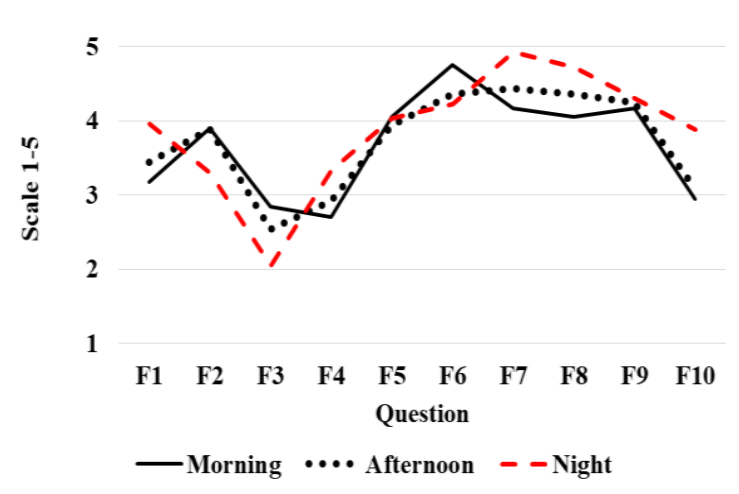

(b)

Figure 2 Questionnaire result of fatigue variables

Figure 2 shows that the average subjective fatigue level for the driving duration of more than five hours is relatively higher compared to a driving duration of less than three hours and three to five hours. However, the fatigue levels during morning, afternoon, and night driving are similar. The questionnaire result for the variable of need for recovery can be seen in Figure 3 below:

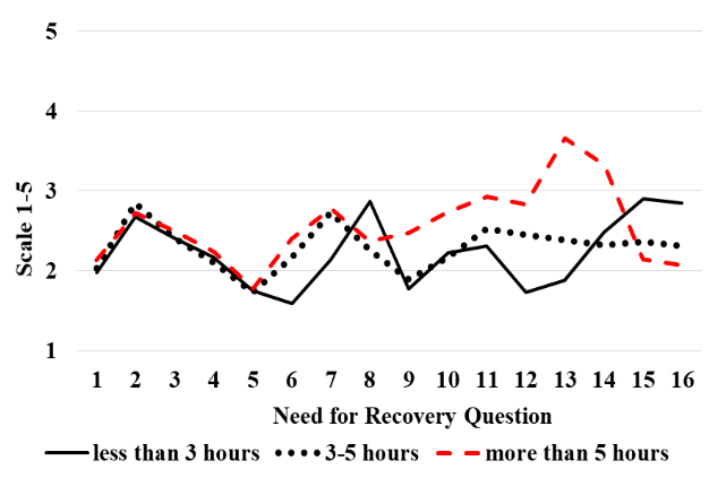

(a)

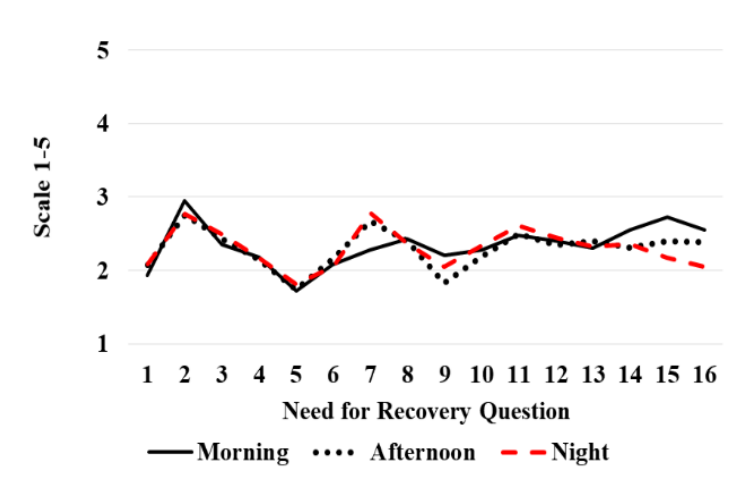

(b)

Figure 3 Questionnaire result of need for recovery variable

The questions for the need for recovery variable were developed to identify resting opportunities for the driver, and the result was used to predict the level of need for recovery. Figure 3 shows that the tendency of the need for recovery is higher for those who 
drive longer than five hours on average. Based on the result grouped by time of day, the tendency for the need for recovery during a morning, afternoon, and night driving schedule is similar (Figure 3). In the following figure, the questionnaire result of EI is plotted (Figure 4).

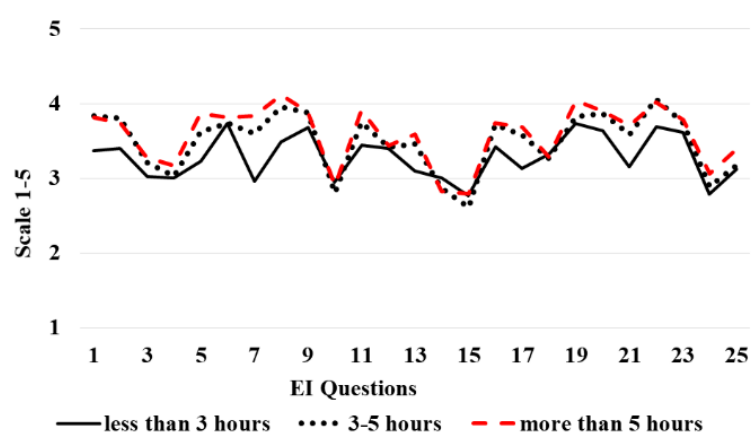

(a)

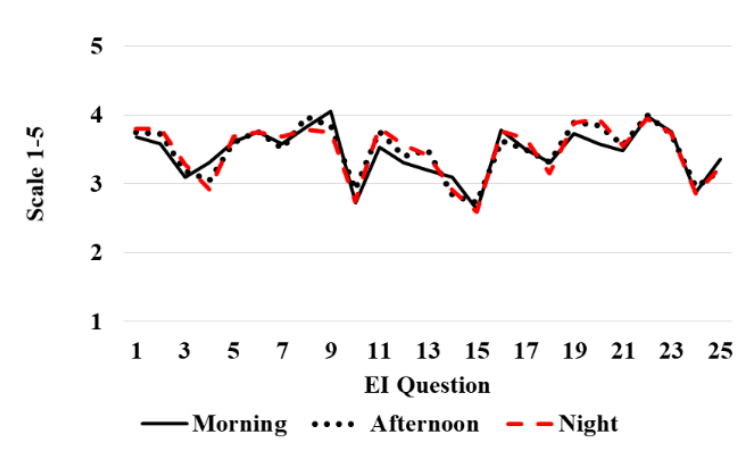

(b)

Figure 4 Questionnaire result of emotional intelligence variable

EI among drivers is similar, as shown in Figure 4. However, those who drive less than three hours generally disagree with the statement in the questionnaire related to the EI variable.

\subsection{Statistical Test Results}

The one-way ANOVA was used to determine whether there are any statistically significant differences of self-perception level for each measure factors among time on task and time of day grouped drivers. The statistical test result showed that there were statistically significant differences in $\mathrm{NR}(\mathrm{F}[2,295]=7.345, p=0.001)$ and $\mathrm{EI}(\mathrm{F}[2,295]=$ $7.095, p=0.001$ ) between the time on task group, while the fatigue level $p$-value resulted in $=0.000$. The test result of the differences based on the time of day group showed that statistically significant differences did not exist (WL: $F[2,295]=1.839, p=0.161$; NR: $F$ $[2,295]=0.230, p=0.795$; EI: $\mathrm{F}[2,295]=1.312, p=0.271)$. A Tukey post hoc test revealed that the NR was statistically significantly higher for a longer duration than three hours $(p=$ 0.025 and $p=0.000$ ). There was no statistically significant difference between the durations of three to five hours and more than five hours of driving ( $p=0.123$ ).

\subsection{Fatigue Related to Workload, Need for Recovery, and Emotional Intelligence}

In this research, the fatigue level was classified into two categories: fatigue (1) and alert (0). Before testing model fits, collected data were labeled as fatigue and alert. In total, 243 drivers were labeled as fatigue and 56 as alert. These labeled samples constituted the use of model fitting and validation. The test result is summarized in Table 1 below.

Table 1 Test result of logistic regression

\begin{tabular}{lrrrccc}
\hline & SE & Sig & Exp. (B) & $\begin{array}{c}\text { Hosmer- } \\
\text { Lemeshow Test Sig. }\end{array}$ & $\begin{array}{c}\text { Cox \& Snell } \\
\text { R-Square }\end{array}$ & $\begin{array}{c}\text { Negelkerke } \\
\text { R-Square }\end{array}$ \\
\hline WL & 0.246 & 0.719 & 1.093 & & & \\
NR & 0.298 & $\mathbf{0 . 0 0 8}$ & 2.211 & $0.673^{*}$ & 0.154 & 0.182 \\
EI & 0.487 & $\mathbf{0 . 0 2 0}$ & 3.091 & & & \\
Constant & -7.426 & 0.000 & 0.001 & & & \\
\hline
\end{tabular}


Binomial logistic regression was run to understand the influence of workload, need for recovery, and EI as subjective fatigue on drivers. The Hosmer-Lemeshow test showed that the model fit the data well, $p=0.678$. Only the need for recovery variable $(p=0.08)$ and EI $(p=0.020)$ statistically significantly predicted subjective fatigue. Based on the result, a mathematical model of logistic regression was written, as follows:

$$
\pi(x)=\frac{\exp (7,426+0,793 \mathrm{NR}+1.129 \mathrm{EI})}{1+\exp (7,426+0,793 \mathrm{NR}+1.129 \mathrm{EI})}
$$

The predicted probabilities are shown graphically, as follows (Figure 5).

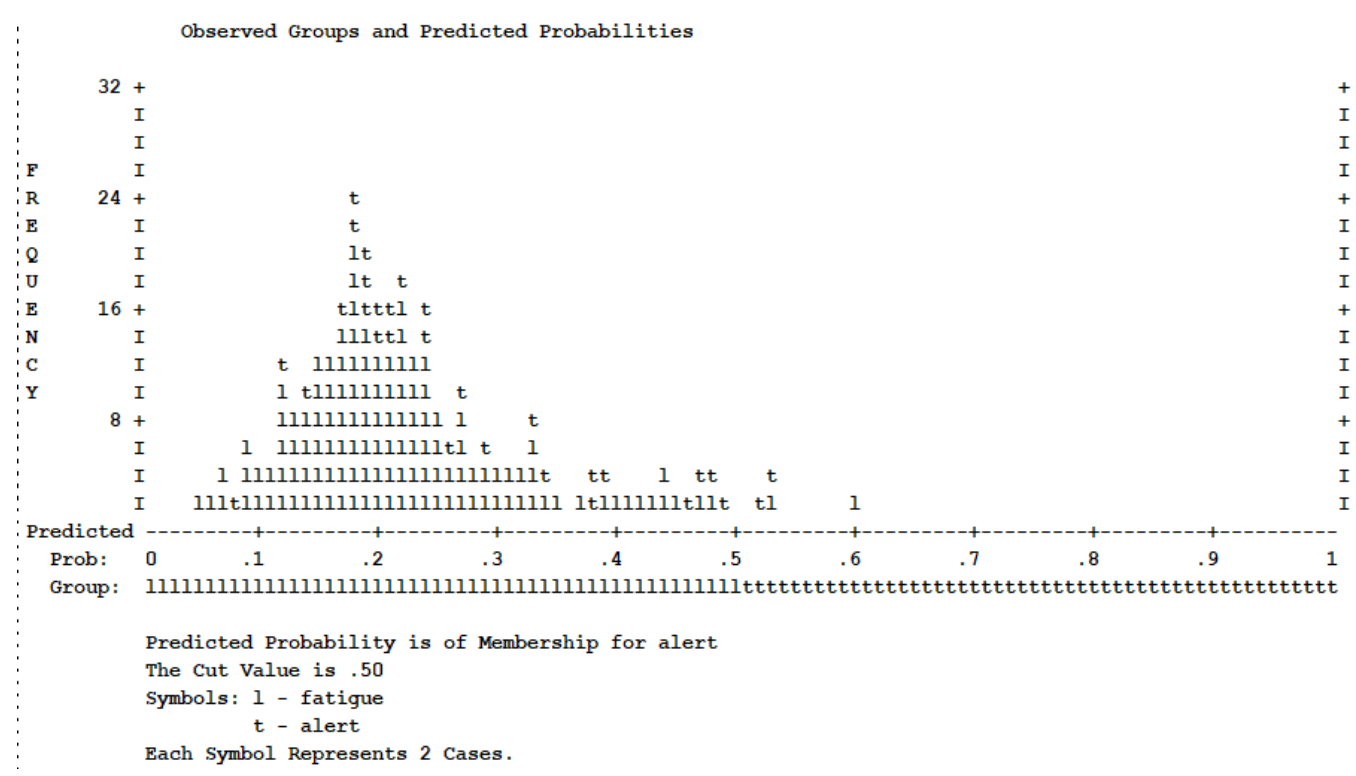

Figure 5 Predicted probabilities of driver fatigue

The left and the right side of the graphic showed the ability of the model to predict the outcome for individual cases. It is represented by a number in which the event of not occurring (indicated by a 'l') was high and the predicted probability of not occurring (indicated by a ' $t$ ') was low. This research, as seen in Figure 4, showed that some cases were in the middle area, and the model identified that the need for recovery and EI were significantly associated with the fatigue outcome. The result indeed could explain $18.29 \%$ of the variance in the outcome (the Nagelkerke pseudo-R2). In this approach, the determinant coefficient indicates that the need for recovery and EI did not fully determine fatigue, although they are significantly associated with it. However, the finding suggested $t$ is an alarm for the driver to prevent fatigue based on recovery needs. The association of EI with fatigue status also suggested that the ability to cope with stress and certain conditions could minimize fatigue exposure to drivers.

\subsection{Discussion}

Many traffic accidents related to driver fatigue are caused by inadequate mental workload, when it is either too low (passive fatigue) or too high (active fatigue) (May and Baldwin, 2009; Gastaldi et al., 2011). In this study, based on the interviews with the drivers, most of the drivers' routes involve toll roads. Thus, the workload is categorized as a monotonous driving-environmental condition. This condition may lead to passive fatigue. However, the workload was perceived as acceptable to drivers. Based on the interviews with the drivers, the acceptance of the condition appeared due to economic reasons. They believe that the workload is one of the consequences of the job; thus, they do not consider it a burden. 
On the contrary, drivers' opportunities to have sufficient rest to be in fit condition to drive are relatively few, as indicated by the result of the NR value, which is moderate to high (3.7 on average scale of one to five). The statistical test showed that the need for recovery for a duration longer than five hours is higher compared to two other driving durations. This result supports other findings that time on task influences the level of driver fatigue (Williamson et al., 2011; Phillips, 2015).

The EI variable as a new perspective proposed in this study showed the ability to differentiate the fatigue and alert conditions of drivers (Table 1). It aligns with the plot of fatigue level in Figure 2a and EI in Figure 4a. The self-awareness, self-regulation, and social skills of drivers are relevant to recognizing their fatigue level. The driving job could be stressful and induce more fatigue in the driver; however, in a stressful situation, such as fatigue from driving, emotionally intelligent individuals show a more adaptive response in coping with the stress (Lea et al., 2019). As seen in Figure 2, drivers perceived the job as mentally fatigued than physical (F2, F4, and F8 were higher compared to F1, F9, and F10). Understanding the situation mentally and having the ability to organize the condition by managing self-acceptance were relevant to the data, thus resulting in the ability to differentiate the fatigue and alert conditions in this study. The result also gives a new point of view that is suitable to improve the safety climate in driving as a job. The need for recovery variable also shows the ability to differentiate the fatigue condition, as expected. This factor was measured to determine how likely driving as a job induced driver fatigue. The correlation among driving duration (NR7-NR-8), rest opportunity (NR9-NR10 and NR14-NR16), and sleep adequacy (NR1-NR3) was measured in this study, and it confirmed the previous result that fatigue results from driving factors.

These findings were important and must be considered to construct a safe driving culture among bus drivers. Improving EI and ensuring sufficient recovery time can help in creating a safety culture among bus drivers and can eliminate unsafe behavior. The safety culture and unsafe conditions have been considered important factors in the transportation system (Mokarami et al., 2019). The (culture is also shaped by how drivers perceive the risk situations they face while driving. There is a negative correlation between risk perception and risk behavior, which means someone would be more careful in their behavior if they had knowledge of the risk (Sutalaksana et al., 2019). With a better EI level, drivers could accept the level of risk in some situations. Safety knowledge should also be considered, as this factor would make drivers more likely to participate in and care about safety improvements (Safitri et al., 2020).

\subsection{Study Limitation and Further Research}

The study has promising findings; however, the workload variable, predicted to have a high correlation with fatigue, was unable to differentiate fatigue conditions, as expected. Other instruments need to be developed in a similar study to verify whether it is true that for intercity bus drivers in Indonesia, the job is categorized as light work and not difficult. For further research, understanding accident risk may provide additional factors to examine how fatigue is perceived by drivers. Understanding the risk is suggested to be added as part of driving safety training programs, as is increased EI training. Recent literature showed that EI can be improved (Gilar-Corb et al., 2019), and it will help drivers to cope with any stress condition that may lead to excessive fatigue and risk behaviors.

To support government programs in reducing road accidents, other research that discusses the deceleration rate, time to collision, and impact speed of buses will be helpful. This kind of research was conducted on motorcycles (da Costa et al., 2018), and ensuring drivers understand these factors (deceleration rate, time to collision and impact speed) in training programs will help reduce accidents significantly. 


\section{Conclusions}

In this research, the discussion of fatigue-related accidents among intercity bus drivers added the EI variable, combined with the workload and need for recovery variables. Subjective workload is perceived as moderate (2.6 on average scale of one to five), while fatigue, NR and EI are moderate to high $(\geq 3.5)$. The most relevant factors related to the fatigue of intercity bus drivers are the need for recovery and EI variables. This study provides a new perspective on the factors influencing fatigue, although there are some limitations in the measurement tools. Any effort to reduce the rate of bus accidents should consider these variables. Therefore, they should be included in safety awareness training among intercity bus drivers.

\section{Acknowledgements}

This study is supported by the Indonesia Ministry of Research, Technology, and Higher Education as part of the Penelitian Unggulan Perguruan Tinggi Research Grant to Binus University with contract number: 225/SP2H/LT/DRPM/2019; 12/AKM/PNT/2019;039/VR.RTT/IV/2019 dated 27 March 2019.

\section{References}

Agresti, A., 2002. Categorical Data. New Jersey: John Wiley and Sons

Bartlett, J.E., Kotrlik, J.Y., Higgins, C.C., 2001. Organizational Research: Determining Appropriate Sample Size in Survey Research. Information Technology, Learning, and Performance Journal, Volume 19(1), pp. 43-50

Chen, C., Zhang, J., 2016. Exploring Background Risk Factors for Fatigue Crashes Involving Truck Drivers on Regional Roadway Networks: A Case Control Study in Jiangxi and Shaanxi, China. Springer Plus, Volume 5(582), pp. 1-12

Craig, A., Tran, Y., Wijesuriya, N., Nguyen, H., 2012. Regional Brain Wave Activity Changes Associated with Fatigue. Psychophysiology, Volume 49(4), pp. 547-582

da Costa, D.G., Malkhamah, S., Suparma, L.B, 2018. Use of the Safety Factor and Margin of Safety in Motorcyclist Accident Risk Management. International Journal of Technology, Volume 9(4), pp. 737-750

Dawson, D., Reynolds, A.C., Van Dongen, H.P., Thomas, M.J., 2018. Determining the Likelihood that Fatigue was Present in a Road Accident: A Theoretical Review and Suggested Accident Taxonomy. Sleep Medicine Reviews, Volume 42, pp. 202-210

De Vries, J., Michielsen, H.J., Van Heck, G.L., 2003. Assessment of Fatigue among Working People: A Comparison of Six Questionnaires. Occupational Environment Medicine, Volume 60(Suppl I), pp. 10-15

Di Milia, L., Smolensky, M., Costa, G., Howarth, H., Ohayon, M., Philip, P., 2011. Demographic Factors, Fatigue, and Driving Accidents: An Examination of the Published Literature. Accident Analysis and Prevention, Volume 43(2), pp. 516-532

Ferguson, S.A., Paech, G., Sargent, C., Darwent, D., Kennaway, D., Roach, G., 2012. The Influence of Circadian Time and Sleep Dose on Subjective Fatigue Ratings. Accident Analysis and Prevention, Volume 45S, pp. 50-54

Friswell, R., Williamson, A., 2013. Comparison of the Fatigue Experiences of Short Haul Night and Long Distance Heavy Vehicle Drivers. Safety Science, Volume 57, pp. 203-213 Gastaldi, M., Rossi, R., Gecchele, G., 2011. Effects of Driver Task-related Fatigue on Driving Performance. Procedia - Social and Behavioral Sciences, Volume 111, pp. 955-964 
Gilar-Corb, R., Pozo-Rico, T., Sánchez, B., Castejón, J.-L., 2019. Can Emotional Intelligence be improved? A Randomized Experimental Study of Business Oriented EI Training Program for Senior Manager. PlosOne, Volume 14(10), pp. 1-21

Hirshkowitz, M., 2013. Fatigue, Sleepiness, and Safety: Definitions, Assessment, Methodology. Sleep Medicine Clinics, Volume 8(2), pp. 183-189

Hosmer, D., Lemeshow, S., 2000. Applied Logistic Regression Second Edition. New York: John Wiley and Sons, Inc.

Jusuf, A., Nurprasetio, I.P., Prihutama, A., 2017. Macro Data Analysis of Traffic Accidents in Indonesia. Journal Engineering Technology Science, Volume 49(1), pp. 132-143

Kulik, A., 2011. Emotional Intelligence and Cumulative Fatigue in Adolescence. Polish Journal of Applied Psychology, Volume 9(1), pp. 7-20

Lea, R.G., Davis, S., Mahoney, B., Qualter, P., 2019. Does Emotional Intelligence Buffer the Effects of Acute Stress? A Systematic Review. Frontiers in Psychology, Volume 10, pp. 119

May, J.F., Baldwin, C.L., 2009. Driver Fatigue: The importance of Identifying Causal Factors of Fatigue. Transportation Research Part F: Traffic Psychology and Behaviour, Volume (12), pp. 218-224

Mohamed, N., Mohd-Yusoff, M.-F., Othman, I., Zulkipli, Z.-H., 2012. Fatigue-related Crashes Involving Express Buses in Malaysia: Will the Proposed Policy of Banning the Earlyhour Operation Reduce Fatigue-related Crashes and Benefit overall Road Safety? Accident Analysis and Prevention, Volume 45, pp. 45-49

Mokarami, H., Shamseddin, S., Pordanjani, T.R., Varmazyar, S., 2019. The Relationship between Organizational Safety Culture and Unsafe Behaviors, and Accidents among Public Transport Bus Drivers using Structural Equation Modeling. Transportation Research Part F: Traffic Psychology and Behaviour, Volume (65), pp. 46-55

Noorbakhsh, S.N., Besharat, M.A., Zarei, J., 2010. Emotional Intelligence and Coping Styles with Stress. Procedia Social and Behavioral Sciences, Volume 5, pp. 818-822

Phillips, R.0., 2015. A Review of Definitions of Fatigue - and a Step Towards a Whole Definition. Transportation Research Part F: Traffic Psychology and Behaviour, Volume (29), pp. 48-56

Polri, 2019. Statistika Laka. (Statistica Laka). Jakarta, DKI Jakarta, Indonesia. Available Online at http://korlantas.polri.go.id/artikel/korlantas/113?Statistik_Laka, Accessed on 20th September 2019

Safitri, D.M., Surjandari, I., Sumabrata, R., 2020. Assessing Factors Affecting Safety Violations of Bus Rapid Transit Drivers in the Greater Jakarta Area. Safety Science, Volume 125, pp. 1-11

Sang, Y., Li, J., 2012. Research on Beijing Bus Driver Psychology Fatigue Evaluation. Procedia Engineering, Volume 43, pp. 443-448

Soehodho, S., 2017. Public Transportation Development and Traffic Accident Prevention in Indonesia. IATSS Research, Volume 40(2), pp. 76-80

Sperandei, S., 2014. Understanding Logistic Regression Analysis. Biochemia Medica, Volume 24(1), pp. 12-18

Sutalaksana, I.Z., Zakiyah, S., Widyanti, A., 2019. Linking Basic Human Values, Risk Perception, Risk Behavior and Accident Rates: The Road to Occupational Safety. International Journal of Technology, Volume 10(5), pp. 918-929

Toomingas, A., Mathiassen, S.E., Tornqvist, E.W., 2012. In: Work, Working Life, Occupational Physiology, Toomingas, A., Mathiassen, S.E., and Tornqvist, E.W., (ed.), Occupational Physiology, pp. 1-18. Boca Raton: CRC Press 
Wedagama, D.M., Wishar, D., 2018. The Relationship between Self-reported Traffic Crashes and Driver Behavior in the Road Transportation of Goods and Freight in Bali. International Journal of Technology, Volume 9(3), pp. 558-567

Williamson, A., Lombardi, D.A., Folkard, S., Stutts, J., Courtney, T.K., Connor, J.L., 2011. The Link between Fatigue and Safety. Accident Analysis and Prevention, Volume 43(2), pp. 498-515

Wulandari, A.P., 2011. Profiling Kecerdasan Emosional Mahasiswa (Student Profiling Emotional Intelligence). Humaniora, Volume 2(1), pp. 190-200 